\title{
Macrophage migration inhibitory factor serves a pivotal role in the regulation of radiation-induced cardiac senescence through rebalancing the microRNA-34a/sirtuin 1 signaling pathway
}

\author{
${\text { YIWANG } \mathrm{HU}^{1 *}, \text { WENZHENG XIA }}^{2 *}$ and MENG HOU ${ }^{3}$ \\ Departments of ${ }^{1}$ Colorectal Surgery, ${ }^{2}$ Neurosurgery and ${ }^{3}$ Radiation Oncology, \\ First Affiliated Hospital, Wenzhou Medical University, Wenzhou, Zhejiang 325000, P.R. China
}

Received May 1, 2018; Accepted August 10, 2018

DOI: $10.3892 /$ ijmm.2018.3838

\begin{abstract}
Radiotherapy significantly increases survival innumerous cancer patients, although it may have delayed adverse effects, including significant short- and long-term effects on cardiovascular function, leading to significant morbidity and mortality. However, the mechanisms underlying these effects remain unclear. Cardiomyocyte senescence contributes to cardiovascular disease via impaired cardiac function. MicroRNA-34a (miR-34a) is a senescence-associated miR involved in the pathology of cardiovascular diseases, while macrophage migration inhibitory factor (MIF) is a cardioprotective cytokine with an important role in cardiovascular diseases. The present study aimed to determine whether MIF has a cytoprotective effect in cardiomyocytes exposed to radiation through modulating miR-34a. Human cardiomyocytes (HCMs) were incubated with MIF and then exposed to radiation. Cellular proliferation was measured using a Cell Counting Kit-8, while cellular senescence was evaluated based on the senescence-associated $\beta$-galactosidase activity and the gene expression levels of cyclin-dependent kinase inhibitor 1a (Cdkn1a) and Cdkn2c. Oxidative stress was evaluated by measuring the generation of reactive oxygen species and malondialdehyde, as well as the expression of antioxidant genes. In addition, HCMs were treated with small interfering RNA against sirtuin 1 (SIRT1) to examine the role of this gene in MIF-associated rejuvenation following radiation-associated senescence. miR-34a was significantly increased in HCMs exposed to radiation, while MIF inhibited senescence by
\end{abstract}

Correspondence to: Dr Meng Hou, Department of Radiation Oncology, First Affiliated Hospital, Wenzhou Medical University, 2 Fuxue Lane, Wenzhou, Zhejiang 325000, P.R. China

E-mail:244517813@qq.com

${ }^{*}$ Contributed equally

Key words: radiation, macrophage migration inhibitory factor, cardiac senescence, microRNA-34a/sirtuin 1 signaling pathway, oxidative stress suppressing miR-34a. SIRT1 was identified as a target gene of miR-34a, mediating the anti-senescence effect induced by MIF. Furthermore, MIF rejuvenation involved rebalancing the oxidation process disturbed by radiation. These results provided direct evidence that inhibition of miR-34a by MIF protected against radiation-induced cardiomyocyte senescence via targeting SIRT1. Inhibition of miR-34a by MIF may thus be a novel strategy for combating cardiac radiation-associated damage.

\section{Introduction}

Randomized trials have reported that radiotherapy can substantially reduce cancer recurrence and moderately reduce cancer mortality; however, cardiac radiation exposure increases the risk of heart failure, thus limiting the use of radiotherapy $(1,2)$. Cardiac radiation exposure in a rodent model resulted in cardiomyocyte hypertrophy, left ventricular diastolic dysfunction, oxidative stress and myocardial fibrosis (3). However, the mechanisms responsible for these effects of radiotherapy remain unclear. A previous study suggested that exposure to radiation affected cardiac physiology-associated gene networks and molecular signaling in cardiomyocytes, with changes in cellular proliferation and cell cycle-associated genes and signaling during the early phase of radiation exposure (4). Furthermore, cardiomyocyte senescence has been demonstrated to be involved in cardiac pathological processes (5). Therefore, the present study aimed to determine whether radiation induced senescence in cardiomyocytes.

MicroRNAs (miRs) are a class of noncoding RNAs that have been reported to serve a significant role in gene regulation by targeting a variety of transcripts. miRs have also recently been identified as important regulators of cellular senescence and aging (6). miR-34a was demonstrated to be involved in the senescence process in cardiomyocytes (7), and exerted a pro-senescence effect by regulating the sirtuin 1 (SIRT1) signaling pathway (8), which serves a crucial role in the maintenance of cardiac homeostasis and energy balance, and is disturbed by radiotherapy (9). Therefore, the current study examined the role of the miR-34a/SIRT1 signaling pathway in radiation-induced cellular senescence. 
Macrophage migration inhibitory factor (MIF) is a multifunctional cytokine secreted by various tissues, including the myocardium (10). Recent evidence has suggested that MIF may have a cardioprotective role in pathological conditions, including ischemia injury, hypertension and cellular senescence (11-13). Several mechanisms have been suggested to account for the MIF-induced biological responses, including modulation of the energy balance, which is known to be disrupted in radiation-induced ischemic heart disease (14). Thus, the role of MIF in the regulation of radiation-induced cellular senescence was also investigated in the present study.

SIRT1, a member of the SIRT family of class III histone deacetylases, is one of the targets of miR-34a, known to be widely involved in the regulation of cellular senescence $(15,16)$. Furthermore, SIRT1 has been reported to be involved in the development and progression of heart failure through the regulation of cell senescence-associated signaling in chemotherapy-induced cardiac damage (17). Consequently, activation of SIRT1 may have a great potential in the treatment of radiation-associated cardiotoxicity.

Oxidative stress is an important inducer of cardiomyocyte senescence (18), with radiation being a major cause of reactive oxygen species (ROS) production (19). Upregulation of antioxidant proteins, such as endothelial nitric oxide synthase (eNOS) and catalase, has been demonstrated to protect against oxidative stress-mediated senescence (20). Growing evidence has also indicated that miR-34a functions asa senescence promoter by inhibiting SIRT1 and inducing oxidative stress (21). Therefore, the present study explored whether radiation-induced oxidative stress exerted a pro-senescence effect in cardiomyocytes.

In the current study, the aim was to examine whether radiation induces cardiomyocyte senescence, and whether MIF reduces cellular susceptibility to radiation-induced senescence by inhibiting miR-34a expression and oxidative stress. The results suggested that MIF may represent a promising therapeutic strategy for radiation-associated cardiac damage.

\section{Materials and methods}

Cell culture and treatment. Human cardiomyocytes (HCMs; PromoCell GmbH, Heidelberg, Germany) were cultured in Dulbecco's modified Eagle's medium (HyClone; GE Healthcare Life Sciences, Logan, UT, USA) supplemented with $10 \%$ fetal calf serum (HyClone; GE Healthcare Life Sciences) and $1 \%$ penicillin-streptomycin (Beyotime Institute of Biotechnology, Jiangsu, China) at $37^{\circ} \mathrm{C}$ with $5 \% \mathrm{CO}_{2}$. In order to induce radiation exposure, $1 \times 10^{5} \mathrm{HCMs}$ growing on 6 -well plates were irradiated in a $15 \times 15 \mathrm{~cm}^{2}$ square field with 5 Gy (6-MeV electron rays; current, $6 \mathrm{~A}$; dose rate, $2 \mathrm{~Gy} / \mathrm{min}$ ), as described previously (22). HCM was treated with $100 \mathrm{ng} / \mathrm{ml}$ human recombinant MIF (cat. no. 289-MF; R\&D Systems, Inc., Minneapolis, MN, USA) for $1 \mathrm{~h}$ at $37^{\circ} \mathrm{C}$.

Cell transfection. HCMs were replated into 6-well plates at a density of $1 \times 10^{5}$ cells per well and then transfected with miR-34a mimic or miR-negative control (NC) mimic (Invitrogen; Thermo Fisher Scientific, Inc., Waltham, MA, USA) using X-tremeGENE ${ }^{\text {TM }}$ HP DNA transfection reagent (Roche Applied Science, Penzberg, Germany) according to the manufacturer's protocol. The miRNA sequences are listed in Table I. Cells were irradiated with 5 Gy, treated with $100 \mathrm{ng} / \mathrm{ml} \mathrm{MIF}$ and harvested $48 \mathrm{~h}$ after transfection for further analysis. The transfection efficiency was analyzed by reverse transcription-quantitative polymerase chain reaction (RT-qPCR).

Small interfering RNA (siRNA) gene knockdown. HMCs $\left(1 \times 10^{5}\right)$ were seeded in 6-well plates at $30-40 \%$ confluence 1 day prior to transfection and reached $70-80 \%$ confluence the following day. Cells were then transfected with $100 \mathrm{nM}$ SIRT1 siRNA and siRNA-non targeting (siRNA-NT; both Invitrogen; Thermo Fisher Scientific, Inc.) using X-tremeGENE HP DNA transfection reagent according to the manufacturer's protocol. The siRNA sequences are listed in Table I. siRNA-NT was used as the control. After a 6 -h incubation at $37^{\circ} \mathrm{C}$, the transfection reagent-siRNA mixture was replaced with fresh growth medium, and the cells were harvested for further experiments at $48 \mathrm{~h}$ after transfection.

Cell proliferation assay. Cell proliferation was assessed using a Cell Counting Kit-8 assay (CCK-8) according to the manufacturer's protocol (Beyotime Institute of Biotechnology). Briefly, cells were seeded at a density of $2 \times 10^{3}$ cells/well in 96-well plates and incubated at $37^{\circ} \mathrm{C}$ for 24,48 and $72 \mathrm{~h}$, respectively. CCK-8 solution $(10 \mu \mathrm{l})$ was added to each well, and the plates were incubated for $1 \mathrm{~h}$ at $37^{\circ} \mathrm{C}$. The absorbance of cells at $450 \mathrm{~nm}$ was then measured with a microplate reader.

$R T$ - $q P C R$. Isolated cells were collected for RT-qPCR analysis. Total RNA was extracted from HCMs using TRIzol reagent (Invitrogen; Thermo Fisher Scientific, Inc.), according to the protocol described by the manufacturer. RNA was then reverse transcribed into cDNA using Transcriptor First Strand cDNA Synthesis kit (Roche Applied Science), followed by analysis by qPCR. The primers used in qPCR for miR-34a, cyclin-dependent kinase inhibitor 1a (Cdkn1a), Cdkn2c, SIRT1, eNOS, catalase and glyceraldehyde-3-phosphate dehydrogenase (GAPDH) are shown in Table II. qPCR was conducted using the FastStart Universal SYBR Green Master (Sigma-Aldrich; Merck KGaA, Darmstadt, Germany) and Applied Biosystems Step One Plus Real-Time PCR system (Thermo Fisher Scientific, Inc.). The thermocycling conditions were as follows: 40 cycles consisting of $95^{\circ} \mathrm{C}$ for $10 \mathrm{sec}, 60^{\circ} \mathrm{C}$ for $10 \mathrm{sec}$ and $72^{\circ} \mathrm{C}$ for $10 \mathrm{sec}$. Quantification was performed relative to the levels of the housekeeping gene GAPDH. Data were analyzed using the $2^{-\Delta \Delta \mathrm{Cq}}$ method (23).

Western blotting. Cells were lysed in ice-cold lysis buffer to obtain the total protein (Beyotime Institute of Biotechnology), and protein concentrations were measured using the BCAProtein Assay kit (Beyotime Institute of Biotechnology). Samples were then separated by $10 \%$ sodium dodecyl sulfate-polyacrylamide gel electrophoresis and blotted onto polyvinylidene fluoride membranes. The membranes were incubated with SIRT1 (cat. no. 9475) and $\beta$-actin (cat. no. 4970) primary antibodies (both 1:1,000; Cell Signaling Technology, Inc., Danvers, MA, USA) overnight, followed by incubation with horseradish peroxidase-conjugated secondary antibodies (cat. no. 7074; 1:2,000; Cell Signaling Technology, Inc.). Subsequently, the membranes were visualized using the BeyoECL Plus (Beyotime Institute 
Table I. Transfected sequences.

\begin{tabular}{ll}
\hline Name & \multicolumn{1}{c}{ Sequence } \\
\hline miR-34a mimic & 5'-UGGCAGUGUCUUAGCUGGUUGUAACCAGCUAAGACACUGCCAUU-3' \\
miR-negative control & 5'-UGUCAGCUUUGGAGCUGGUUGUAACCUAAGAUGCCACCAGCAUU-3' \\
siRNA-Sirtuin 1 & F: 5'-AAGTACAATCCACTCCGGAA TGA-3' \\
siRNA-non targeting & F: 5'-CTCTATGAACACCTAGCT-3'
\end{tabular}

miR, microRNA; NC, si, small interfering; F, forward

Table II. Primer sequences used in quantitative polymerase chain reaction.

\begin{tabular}{ll}
\hline Gene & \multicolumn{1}{c}{ Sequence } \\
\hline microRNA-34a & F: 5'-CAGAGCATCACACGCAAGC-3' \\
Cdkn1a & R: 5'-CAGGAAACAGAAACCCCAGC-3' \\
& F: 5'-TCACTGTCTTGTACCCTTGTGC-3' \\
Cdkn2c & R: 5'-GGCGTTTGGAGTGGTAGAAA-3' \\
& F: 5'-CGGGAGGTTCTTGTTCTG-3' \\
Sirtuin 1 & R: 5'-TTTGTTGGCTTGCTTGAC-3' \\
& F: 5'-CAGAGCAT CACACGCAAGC-3' \\
Telomere length & R: 5'-CAGGAAACAG AAACCCCAGC-3' \\
& F: 5'-CGGTTTGTTTGGGTTTGGGTTTGGGTTTGGGTTTGGGTT-3' \\
Endothelial nitric oxide synthase & R: 5'-GGCTTGCCTTACCCTTACCCTTACCCTTACCCTTACCCT-3' \\
& F: 5'-GCAACCACATCAAGTATGCCACCAA-3' \\
Catalase & R: 5'-GCTGTTCCAGATTCGGAAGTCTCCT-3' \\
& F: 5'-CAAGCTGGTTAATGCGAATGG-3' \\
GAPDH & R: 5'-TTGAAAAGATCTCGGAGGCC-3' \\
& F: 5'-CTGCACCACCAACTGCTTAG-3' \\
& R: 5'-AGGTCCACCACTGACACGTT-3'
\end{tabular}

miR, microRNA; Cdkn, cyclin-dependent kinase inhibitor; F, forward; R, reverse.

of Biotechnology). The stained protein bands were visualized using a Bio-RadChemiDoc XRS system and analyzed using Quantity One software (Bio-Rad Laboratories, Inc., Hercules, CA, USA).

Relative telomere length. The relative telomere length in HCMs was measured using a qPCR approach, as described previously (24), with GAPDH serving as the normalizing gene. Table II lists the primers used for the detection of the telomere length.

Relative telomerase activity. A TeloTAGGG Telomerase PCR ELISA PLUS Assay kit (Sigma-Aldrich; Merck KGaA, Darmstadt, Germany) was used to analyze the relative telomerase activity, according to the protocol provided by the manufacturer.

Senescence-associated $\beta$-galactosidase (SA- $\beta$-gal) assay. Cellular senescence was measured by SA- $\beta$-gal assay (Cell Signaling Technology, Inc., Danvers, MA, USA). Briefly, cells were seeded in a 6 -well plate at a density of $2 \times 10^{4}$ cells/well were washed with phosphate-buffered saline, fixed with $2 \%$ paraformaldehyde for $30 \mathrm{~min}$ at room temperature and then incubated with fresh SA- $\beta$-gal staining solution, as described previously (25). SA- $\beta$-gal activity was measured using a microplate reader at a wavelength of $50 \mu \mathrm{m}$.

ROS measurement. Intracellular ROS levels were measured using 2,7-dichlorodihydrofluorescein diacetate (Beyotime Institute of Biotechnology), according to the protocol described by the manufacturer. A fluorescence spectrophotometer was used to determine the fluorescence intensity of the cells, at an excitation and emission wavelength of 488 and $525 \mathrm{~nm}$, respectively.

Lipid peroxidation assays. Malondialdehyde (MDA) levels in HMCs were measured using the Lipid Peroxidation (MDA) Assay kit (Abcam, Cambridge, UK). Briefly, 2 ml HMCs at a density of $1 \times 10^{6}$ cells $/ \mathrm{ml}$ were homogenized on ice in $300 \mu \mathrm{l}$ MDA lysis buffer, followed by centrifugation at 12,000 $\mathrm{x} g$ and 

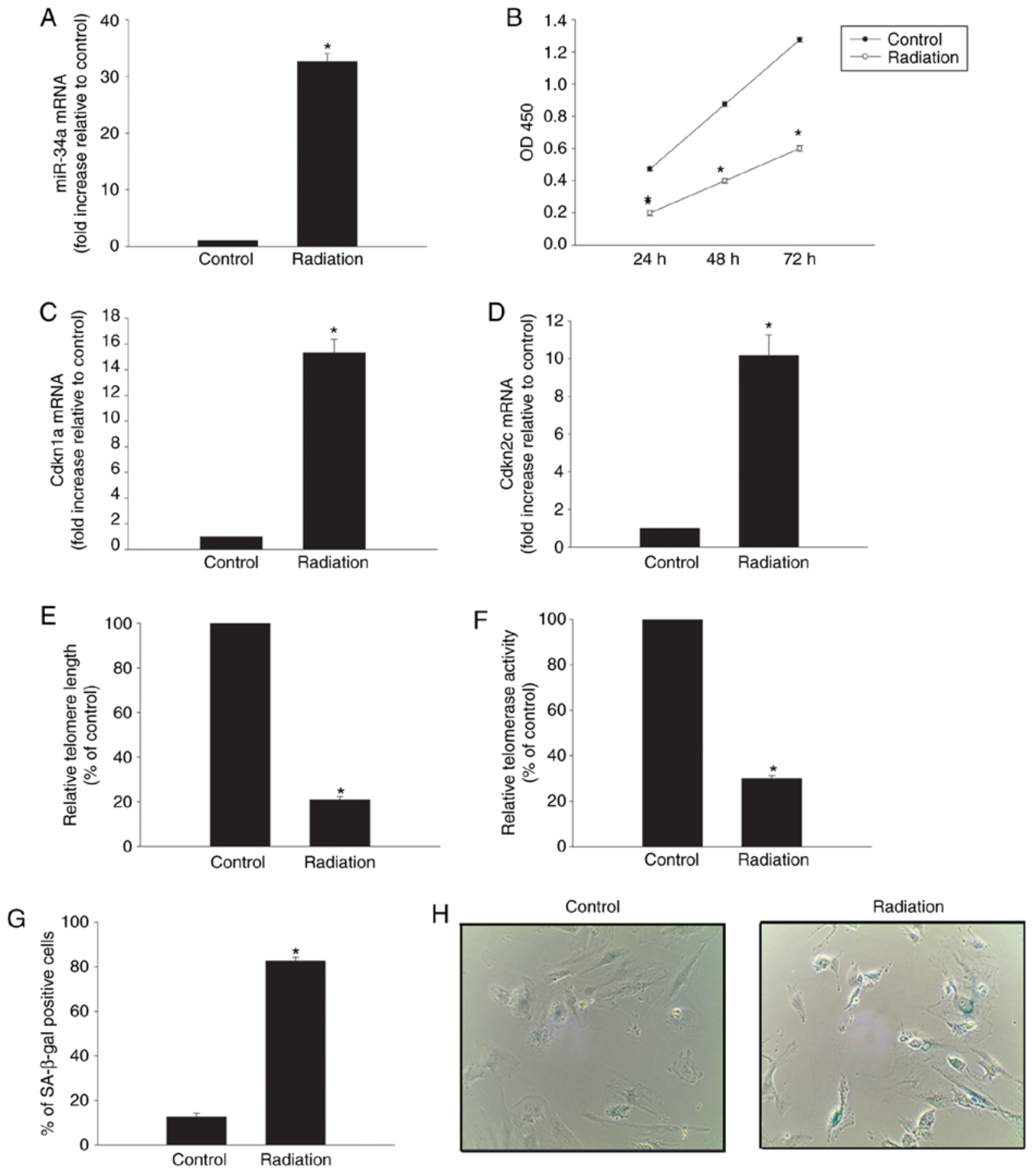

Figure 1. Radiation induces miR-34a expression and cellular senescence in HCMs. (A) miR-34a expression levels analyzed by RT-qPCR. (B) Cell proliferation curves determined by Cell Counting Kit-8 assay. (C) Cdkn1a, (D) Cdkn2c and (E) relative telomere length mRNA levels were analyzed by RT-qPCR. (F) Relative telomerase activity was measured. (G) Percentage of SA- $\beta$-gal-positive cells, and (H) representative images of SA- $\beta$-gal staining. Each column represents the mean \pm standard deviation from three independent experiments. "P<0.05 vs. control group. miR, microRNA; RT-qPCR, reverse transcription-quantitative polymerase chain reaction; $\mathrm{Cdkn}$, cyclin-dependent kinase inhibitor; SA- $\beta$-gal, senescence-associated $\beta$-galactosidase.

$4^{\circ} \mathrm{C}$ for $1 \mathrm{~min}$ to remove insoluble material. Thiobarbituric acid was then added to the supernatant and incubated at $95^{\circ} \mathrm{C}$ for $60 \mathrm{~min}$. The samples were allowed to cool at room temperature for $10 \mathrm{~min}$, and the absorbance at $532 \mathrm{~nm}$ was measured with a spectrophotometer.

Statistical analysis. Data are expressed as the mean \pm standard deviation. Differences among groups were tested by one-way analysis of variance, and comparisons between two groups were evaluated by Student's t-tests using SPSS version 19.0 software (IBM Corporation, Armonk, NY, USA). A value of $\mathrm{P}<0.05$ was considered to denote a difference that was statistically significant.

\section{Results}

Radiation induces miR-34a expression and senescence in HCMs . miR-34a expression levels in HCMs were significantly increased following exposure to radiation (Fig. 1A). Radiation markedly inhibited cellular proliferation 

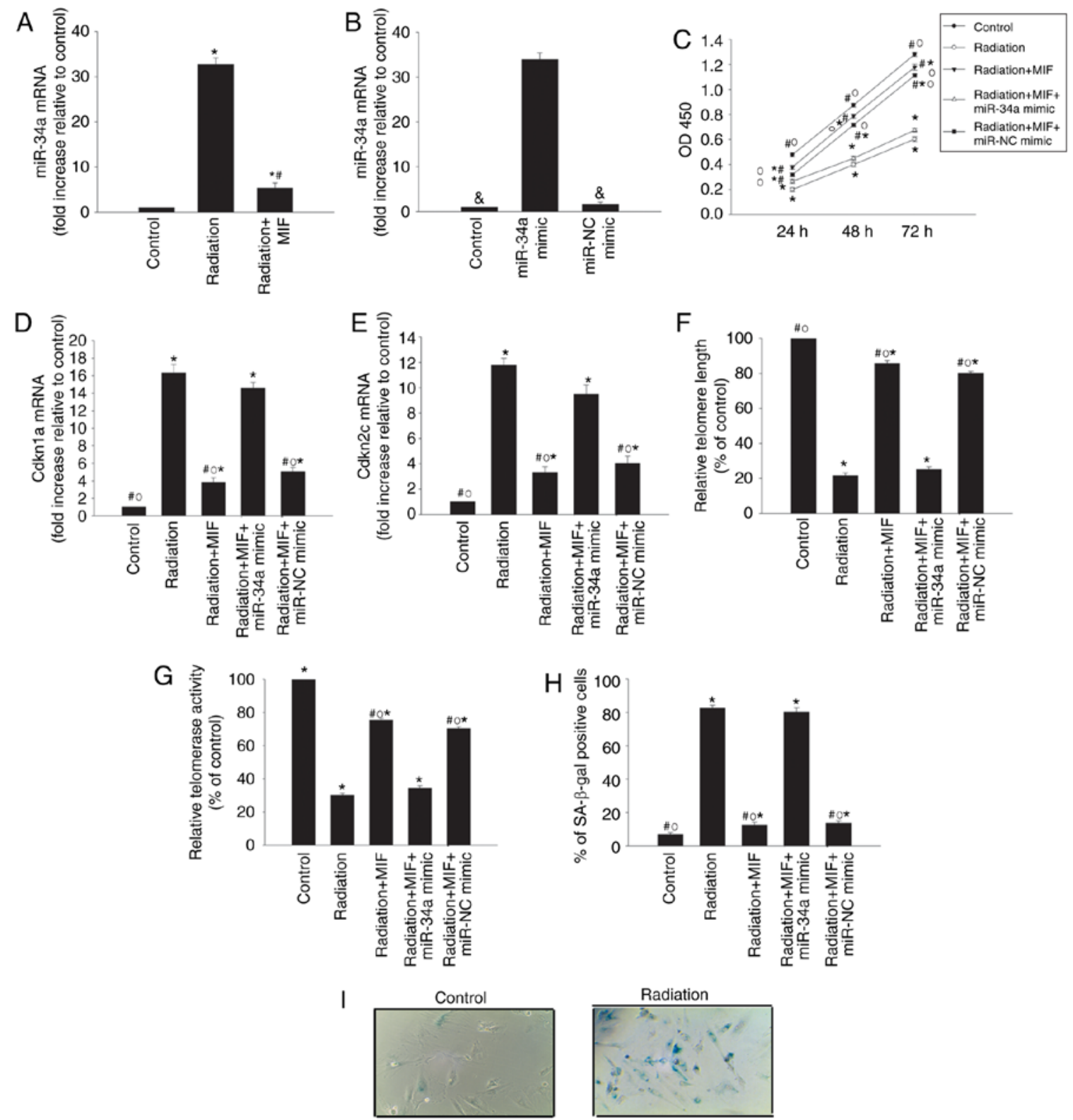

Radiation
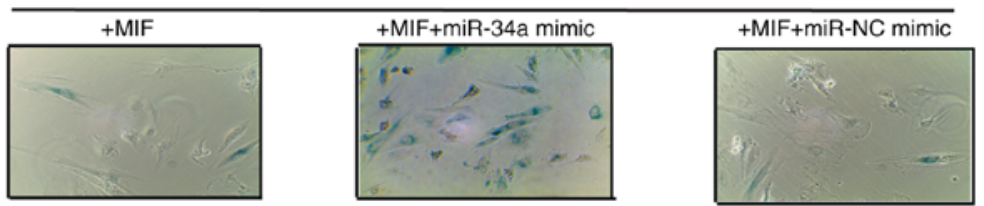

Figure 2. Exogenous MIF alleviates radiation-induced senescence via inhibition of miR-34a. (A) miR-34a expression levels in control and radiation-exposed cardiomyocytes with or without MIF treatment $(100 \mathrm{ng} / \mathrm{ml})$, as determined by RT-qPCR. (B) miR-34a expression levels in untransfected cardiomyocytes, and cardiomyocytes transfected with miR-34a mimic or miR-NC mimic, as determined by RT-qPCR. (C) Cell growth curves determined by Cell Counting Kit-8 assay in cardiomyocytes transfected with miR-34a mimic or miR-NC mimic, pretreated with MIF and exposed to radiation. Untreated cardiomyocytes serve as the control. (D) Cdkn1a, (E) Cdkn2c and (F) relative telomere length mRNA levels were analyzed by RT-qPCR. (G) Relative telomerase activity. (H) Percentage of SA- $\beta$-gal-positive cells, and (I) representative images of SA- $\beta$-gal staining. Data represent the mean \pm standard deviation from three independent experiments. ${ }^{*} \mathrm{P}<0.05$ vs. control group; ${ }^{\#} \mathrm{P}<0.05$ vs. radiation group; ${ }^{\&} \mathrm{P}<0.05$ vs. miR-NC group; ${ }^{\circ} \mathrm{P}<0.05$ vs. radiation $+\mathrm{MIF}+\mathrm{miR}-34 \mathrm{a}$ mimicgroup. MIF, macrophage migration inhibitory factor; miR, microRNA; NC, negative control; RT-qPCR, reverse transcription-quantitative polymerase chain reaction; Cdkn, cyclin-dependent kinase inhibitor; SA- $\beta$-gal, senescence-associated $\beta$-galactosidase.

(Fig. 1B) and increased the expression levels of the cellular senescence-associated genes Cdkn1a and Cdkn2c (Fig. 1C and D). Radiation exposure also significantly shortened the relative telomere length (Fig. 1E), impaired the relative telomerase activity (Fig. 1F) and increased the percentage of SA- $\beta$-gal-positive cells (Fig. $1 \mathrm{G}$ and $\mathrm{H}$ ).
Exogenous MIF alleviates radiation-induced senescence via inhibition of $\mathrm{miR}-34 \mathrm{a}$. The anti-senescence effect of MIF was explored by adding $100 \mathrm{ng} / \mathrm{ml}$ MIF to HCMs prior to exposure to radiation. Exogenous MIF significantly decreased the expression of miR-34a induced by radiation (Fig. 2A). Subsequently, the study further examined whether MIF 
exerted its anti-senescence effect via inhibition of miR-34a by transfecting HCMs with a miR-34a mimic to induce the overexpression of miR-34a (Fig. 2B). MIF treatment in cells exposed to radiation increased cellular proliferation (Fig. 2C), decreased the expression levels of the senescence-associated genes Cdkn1a and Cdkn2c (Fig. 2D and E), recovered the impaired relative telomere length and activity (Fig. $2 \mathrm{~F}$ and G), and reduced the percentage of SA- $\beta$-gal-positive cells (Fig. $2 \mathrm{H}$ and I). However, the effects of MIF treatment were reversed by miR-34a overexpression in cells exposed to radiation.

Rebalancing of the miR-34a/SIRT1 signaling pathway by MIF is essential for preventing radiation-associated senescence. SIRT1 is as a well-known target of miR-34a and is closely associated with cellular senescence (8). The present study analyzed SIRT1 expression to investigate the miR-34a target and clarify the mechanism of MIF-mediated suppression of radiation-associated cellular senescence in HCMs. SIRT1 protein expression was significantly inhibited by radiation and then recovered by MIF; however, the MIF-mediated recovery was reversed by miR-34a overexpression (Fig. 3A and B). Furthermore, the mechanism responsible for the anti-senescence effect of MIF in relation to the miR-34a/SIRT1 signaling pathway was further explored by silencing SIRT1 using siRNA (Fig. 3C). Downregulation of SIRT1in MIF-treated radiation-exposed cells decreased the recovery of cell proliferation induced by MIF (Fig. 4A), induced the expression of the senescence-associated genes Cdkn1a and Cdkn2c (Fig. 4B and C), reduced the telomere length and activity (Fig. 4D and $\mathrm{E}$ ), and increased the percentage of SA- $\beta$-gal-positive cells (Fig. 4F and G), as compared with the cells treated with only MIF and radiation. By contrast, none of these effects were observed in HCMs treated with the control, siRNA-NT.

Modulation of oxidative stress by MIF inhibits the radiation-induced senescence. The effects of MIF on oxidants and antioxidant gene expression levels were assessed to determine whether MIF exerted its anti-senescence effect via modulation of oxidative stress in cardiomyocytes. Quantitative analysis demonstrated that MIF inhibited the radiation-induced accumulation of cellular ROS and generation of MDA in HCMs (Fig. 5A and B), and reversed the radiation-mediated inhibition of eNOS and catalase gene expression levels (Fig 5C and D). These antioxidant effects of MIF were abolished by overexpression of miR-34a or silencing of SIRT1 (Fig. 5).

\section{Discussion}

Recent progress in radiotherapy over the past two decades has led to substantial improvements in life expectancy for cancer patients, however, at the cost of increased risks of unintended side effects (26). Radiotherapy has been closely associated with cardiotoxicity and an increased risk of symptomatic cardiac dysfunction $(1,27)$. Given the increasing number of long-term survivors following treatment with radiotherapy, cardiotoxicity has become recognized as a major concern in oncology (28). However, the mechanisms responsible for radiotherapy-associated cardiac damage remain unclear. Heart aging is associated with functional decline and increased vulnerability
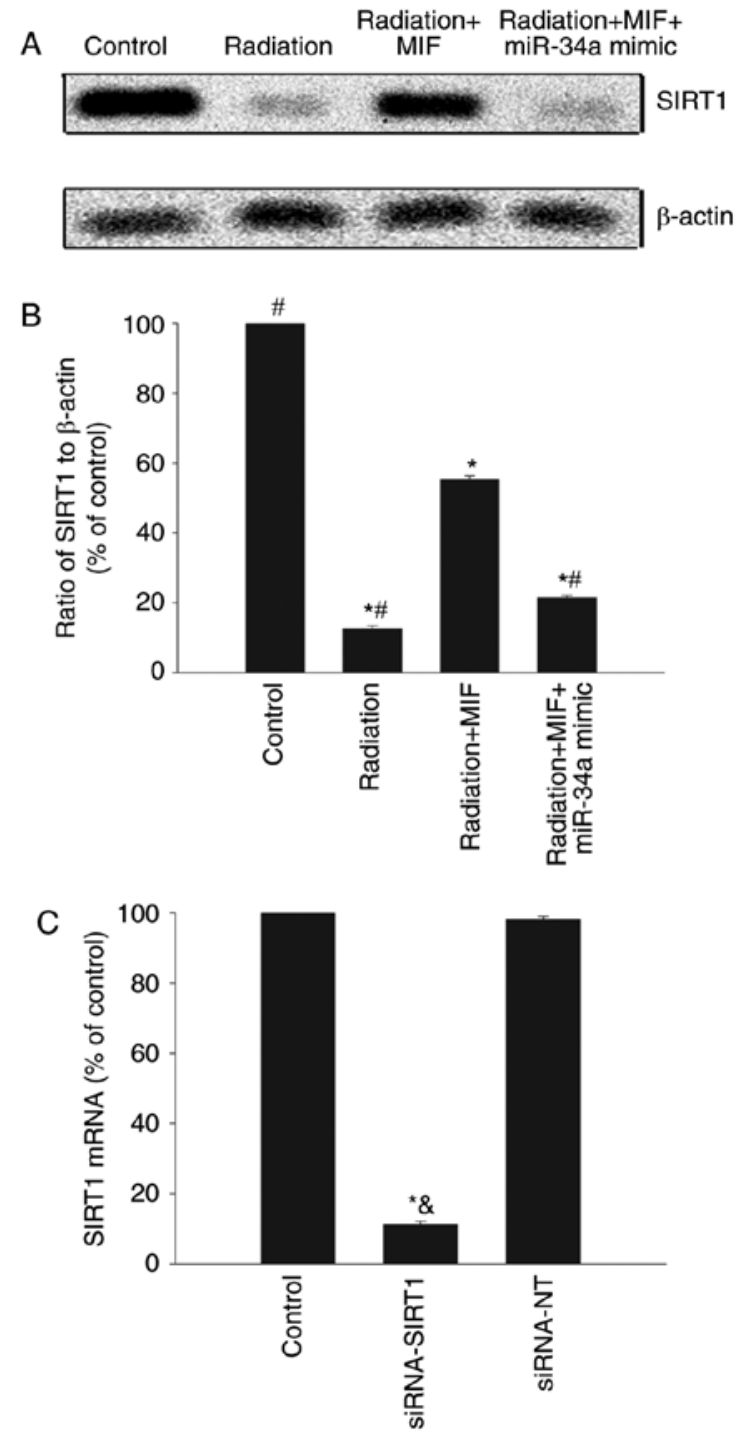

Figure 3. MIF rebalances the miR-34a/SIRT1 signaling pathway. (A) Western blot images and (B) quantified results of SIRT1 protein expression levels in cardiomyocytes with or without transfection with miR-34a mimic, pretreated with MIF and exposed to radiation. (C) Cardiomyocytes were transfected with siRNA against SIRT1 or with siRNA-NT, serving as the control. siRNA-mediated transfection efficiency was determined by reverse transcription-quantitative polymerase chain reaction. Data represent the mean \pm standard deviation of three independent experiments. ${ }^{*} \mathrm{P}<0.05$ vs control group; ${ }^{\#} \mathrm{P}<0.05$ vs. radiation + MIF group; ${ }^{\&} \mathrm{P}<0.05$ vs. siRNA-NT group. MIF, macrophage migration inhibitory factor; miR, microRNA; siRNA, small interfering RNA; SIRT1, sirtuin 1.

to cardiovascular damage, and is the leading cause of mortality worldwide (29). Age-associated changes include decreased cardiac output, elevated susceptibility to arrhythmia and impaired relaxation with increased myocardial stiffness, all of which are included in the phenotype of radiotherapy-associated cardiac damage $(1,30)$. The results of the current study revealed that radiation induced senescence in HCMs.

miRs have emerged as a new class of modulators of gene expression. Among these, miR-34a has been implicated in cardiac-associated damage, particularly cardiac senescence (31). Researchers recently demonstrated that miR-34a was involved in modulating radiation-induced senescence, and that radiation-induced senescence was correlated with the upregulation of miR-34a expression (32). Accordingly, 

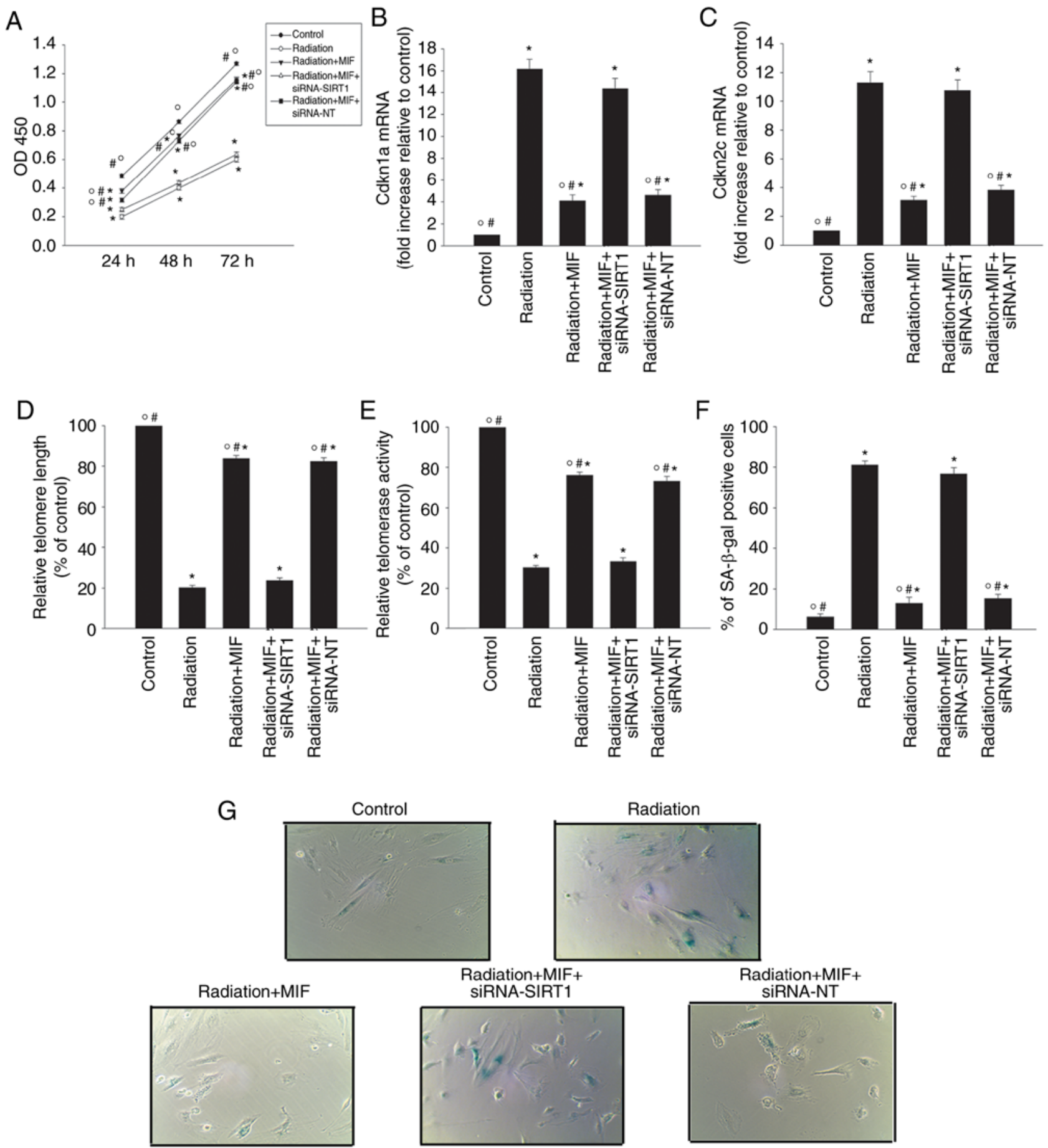

Figure 4. Rebalancing the miR34a/SIRT1 signaling pathway induced by MIF is essential for preventing radiation-associated senescence. Cardiomyocytes were transfected with siRNA-SIRT1 or siRNA-NT, pretreated with MIF and exposed to radiation. Untreated cardiomyocytes were used as the control. (A) Cell growth curves were determined by the Cell Counting Kit-8 assay. (B) Cdkn1a, (C) Cdkn2c and (D) relative telomere length mRNA levels were analyzed by reverse transcription-quantitative polymerase chain reaction. (E) Relative telomerase activity. (F) Percentage of SA- $\beta$-gal-positive cells, and (G) representative images of SA- $\beta$-gal staining. Data represent the mean \pm standard deviation from three independent experiments. ${ }^{*} \mathrm{P}<0.05$ vs.control group; ${ }^{*} \mathrm{P}<0.05$ vs. radiation group; ${ }^{\circ} \mathrm{P}<0.05$ vs. radiation+MIF+siRNA-SIRT1group. MIF, macrophage migration inhibitory factor; miR, microRNA; siRNA, small interfering RNA; SIRT1, sirtuin 1; Cdkn, cyclin-dependent kinase inhibitor; SA- $\beta$-gal, senescence-associated $\beta$-galactosidase.

the present study found that radiation exposure induced miR-34a expression in HCMs, accompanied by cellular senescence.

MIF is known as a prototype cytokine that regulates macrophage function in inflammation and serves an important role in cardiac metabolism (33). Previously, it was confirmed that MIF was highly expressed in cardiomyocytes and regulated cellular senescence (10). As a promoter of miR-34a, p53 is associated with accelerated cellular senescence and is inhibited by MIF $(34,35)$. The results of the present study revealed that MIF alleviated radiation-associated cellular senescence through inhibiting miR-34a expression, while overexpression of miR-34a abolished the anti-senescence effect of MIF. miR-34a has been reported to trigger senescence 

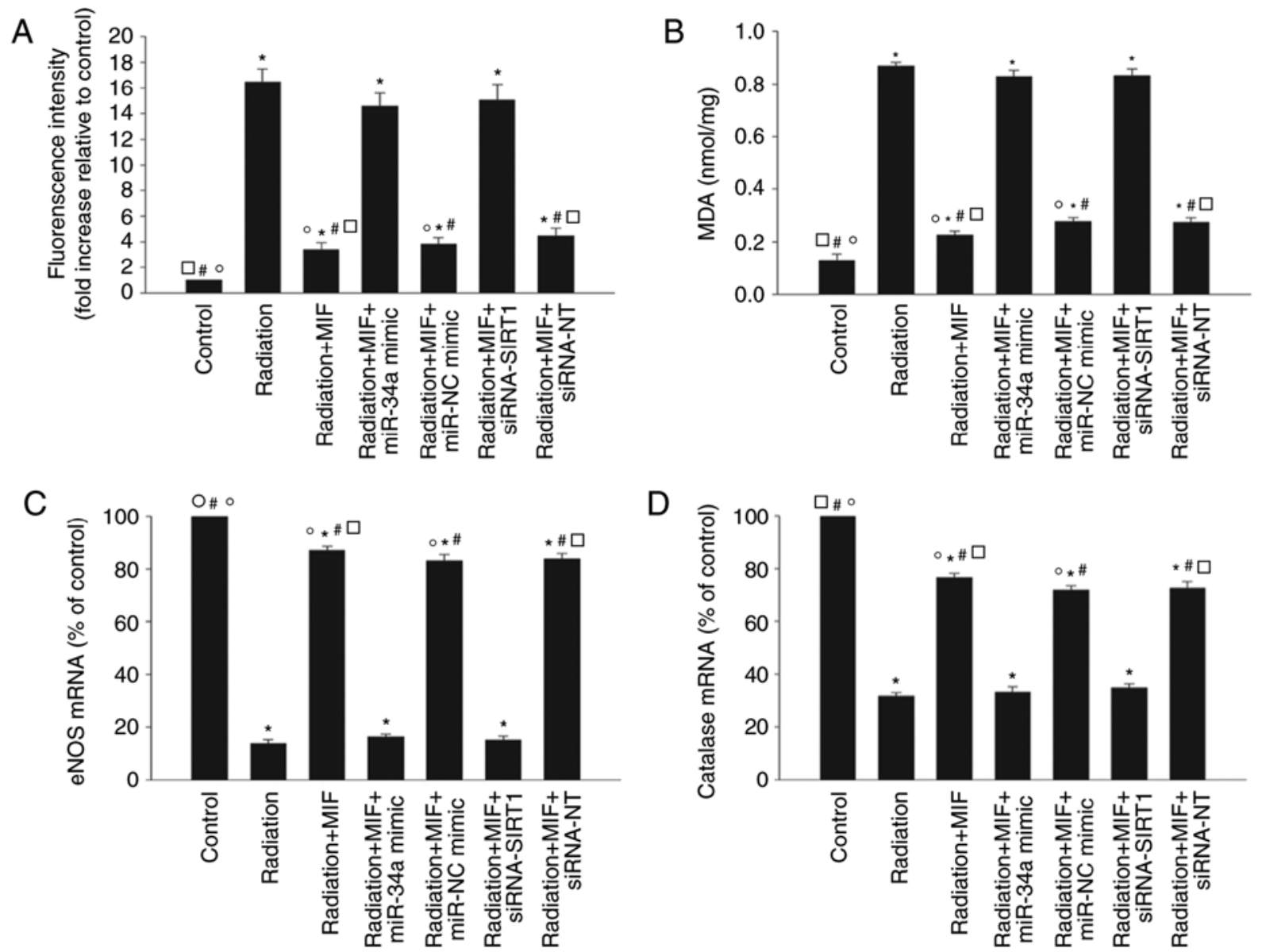

Figure 5. Modulation of oxidant stress by MIF inhibits radiation-induced senescence. Cardiomyocytes were transfected with miR-34a mimic, miR-NC mimic, siRNA-SIRT1 or siRNA-NT, then pretreated with MIF and exposed to radiation. Untreated cardiomyocytes were used as the control. (A) Intracellular ROS production analyzed by fluorescence spectrophotometry. (B) Lipid peroxidation evaluated by MDA formation. (C) eNOS and (D) catalase mRNA levels measured by reverse transcription-quantitative polymerase chain reaction. Each column represents the mean \pm standard deviation from three independent experiments. ${ }^{*} \mathrm{P}<0.05$ vs. control group; ${ }^{\sharp} \mathrm{P}<0.05$ vs. radiation group; ${ }^{\circ} \mathrm{P}<0.05$ vs. radiation+MIF+miR-34a mimic group; ${ }^{\square} \mathrm{P}<0.05$ vs. radiation+MIF+siRNA-SIRT1 group. MIF, macrophage migration inhibitory factor; miR, microRNA; NC, negative control; siRNA, small interfering RNA; SIRT1, sirtuin 1; ROS, reactive oxygen species; MDA, malondialdehyde; eNOS, endothelial nitric oxide synthase.

partly through genetic inhibition of SIRT1 (36); therefore, this observation prompted us to explore the potential role of MIF in modulating miR-34a-dependent SIRT1 expression in cardiomyocytes. It was observed that MIF significantly suppressed the radiation-induced expression of miR-34a, while silencing SIRT1 blocked the anti-senescence effects of MIF. These results revealed that MIF reduced cardiomyocyte senescence by interacting with miR-34a and upregulating SIRT1.

Cellular senescence is defined as the stable cell cycle arrest elicited in response to a variety of stressors (37). The accumulation of oxidative stress in cardiomyocytes has been reported to cause senescence and reduce cardiac function (18). Radiation-associated oxidative stress and telomere loss have all been linked to the induction of the senescent phenotype through replication stress-induced DNA damage $(38,39)$. The current study demonstrated that cardiomyocyte senescence was accompanied by radiation-induced oxidative stress and telomere loss. MIF has been reported to function as an antioxidant factor (40). Accordingly, the present study found that MIF significantly inhibited ROS and MDA generation, and stimulated eNOS and catalase gene expression in cardiomyocytes. Furthermore, overexpression of miR-34a by transfection of amiR-34a mimic or silencing SIRT1 abolished the MIF-induced antioxidant gene expression.

In conclusion, the results of the present study demonstrated that MIF had a protective role in radiation-induced cardiac senescence. This cardioprotective effect of MIF may mainly be attributed to the suppression of oxidative stress by preventing miR-34a-mediated inhibition of antioxidant gene expression. This increased understanding of the complex signaling networks involved in radiation-associated cardiac injuries will help to establish methods to alleviate cardiac damage induced by radiation.

\section{Acknowledgements}

Not applicable.

\section{Funding}

The current study was supported by the National Natural Science Foundation of China (grant nos. 81500261 and 
81600278 to $\mathrm{MH}$ and WX, respectively) and the Medical Science and Technology Project of Zhejiang Province (grant no. 2018KY517 to MH).

\section{Availability of data and materials}

All data generated or analyzed during the present study are included in the published article.

\section{Authors' contributions}

YH and WX made substantial contributions to the acquisition, analysis and interpretation of data. MH was involved in conception and design of the study, and in drafting the manuscript. All authors read and approved the final manuscript

\section{Ethics approval and consent to participate}

All animal procedures were approved by the Institutional Animal Care and Use Committee of Wenzhou Medical University.

\section{Patient consent for publication}

Not applicable.

\section{Competing interests}

The authors declare that they have no competing interests.

\section{References}

1. Taylor C, Correa C, Duane FK, Aznar MC, Anderson SJ, Bergh J, Dodwell D, Ewertz M, Gray R, Jagsi R, et al: Estimating the risks of breast cancer radiotherapy: Evidence from modern radiation doses to the lungs and heart and from previous randomized trials. J Clin Oncol 35: 1641-1649, 2017.

2. EBCTCG (Early Breast Cancer Trialists' Collaborative Group), McGale P, Taylor C, Correa C, Cutter D, Duane F, Ewertz M, Gray R, Mannu G, Peto R, et al: Effect of radiotherapy after mastectomy and axillary surgery on 10-year recurrence and 20-year breast cancer mortality: Meta-analysis of individual patient data for 8135 women in 22 randomised trials. Lancet 383 : 2127-2135, 2014.

3. Saiki H, Moulay G, Guenzel AJ, Liu W, Decklever TD, Classic KL, Pham L.Chen HH, Burnett JC, Russell SJ and Redfield MM: Experimental cardiac radiation exposure induces ventricular diastolic dysfunction with preserved ejection fraction. Am J Physiol Heart Circ Physiol 313: H392-H407, 2017.

4. Coleman MA, Sasi PS, Onufrak J, Natarajan M, Manickam K, Schwab J, Muralidharan S, Peterson LE, Alekseyev YO, Yan X and Goukassian DA: Low dose radiation affects cardiac physiology: Gene networks and molecular signaling in cardiomyocytes. Am J Physiol Heart Circ Physiol 309: H1947-H1963, 2015.

5. Du WW, Yang W, Chen Y, Wu ZK, Foster FS, Yang Z, Li X and Yang BB: Foxo3 circular RNA promotes cardiac senescence by modulating multiple factors associated with stress and senescence responses Eur Heart J 38: 1402-1412, 2017.

6. Smith-Vikos T and Slack FJ: MicroRNAs and their roles in aging. J Cell Sci 125: 7-17, 2012.

7. Yang Y, Cheng HW, Qiu Y, Dupee D, Noonan M, Lin YD, Fisch S, Unno K, Sereti KI and Liao R: MicroRNA-34a plays a key role in cardiac repair and regeneration following myocardial infarction. Circ Res 7: 450-459, 2015.

8. Fomisonnurse I, Saw EEL, Gandhi S, Munasinghe PE, Van Hout I, Williams MJA, Galvin I, Bunton R, Davis P, Cameron V and Katare R: Diabetes induces the activation of pro-ageing miR-34a in the heart, but has differential effects on cardiomyocytes and cardiac progenitor cells. Cell Death Differ 25: 1336-1349, 2018.
9. Hong EH, Lee SJ, Kim JS, Lee KH, Um HD, Kim JH, Kim SJ, Kim JI and Hwang SG: Ionizing radiation induces cellular senescence of articular chondrocytes via negative regulation of SIRT1 by p38 kinase. J Biol Chem 285: 1283-1295, 2010.

10. Miller EJ, Li J, Leng L, McDonald C, Atsumi T, Bucala R and Young LH: Macrophage migration inhibitory factor stimulates AMP-activated protein kinase in the ischaemic heart. Nature 451: 578-582, 2008.

11. Ma H, Wang J, Thomas DP, Tong C, Leng L, Wang W, Merk M, Zierow S, Bernhagen J, Ren J, et al: Impaired macrophage migration inhibitory factor-AMP-activated protein kinase activation and ischemic recovery in the senescent heart. Circulation 122: 282-292, 2010.

12. Xu X, Hua Y, Nair S, Bucala R and Ren J: Macrophage migration inhibitory factor deletion exacerbates pressure overload-induced cardiac hypertrophy through mitigating autophagy. Hypertension 63: 490-499, 2014.

13. Xu X, Pacheco BD, Leng L, Bucala R and Ren J: Macrophage migration inhibitory factor plays a permissive role in the maintenance of cardiac contractile function under starvation through regulation of autophagy. Cardiovasc Res 99: 412-421, 2013.

14. Azimzadeh O, Azizova T, Merlpham J, Subramanian V, Bakshi MV, Moseeva M, Zubkova O, Hauck SM, Anastasov N, Atkinson MJ and Tapio S: A dose-dependent perturbation in cardiac energy metabolism is linked to radiation-induced ischemic heart disease in Mayak nuclear workers. Oncotarget 8: 9067-9078, 2017.

15. Haigis MC and Sinclair DA: Mammalian sirtuins: Biological insights and disease relevance. Annu Rev Pathol 5: 253-295, 2009.

16. Morris BJ: Seven sirtuins for seven deadly diseases of aging. Free Radic Biol Med 56: 133-171, 2013.

17. De Angelis A, Piegari E, Cappetta D, Russo R, Esposito G, Ciuffreda LP, Ferraiolo FA, Frati C, Fagnoni F, Berrino L, et al: SIRT1 activation rescues doxorubicin-induced loss of functional competence of human cardiac progenitor cells. Int J Cardiol 189: 30-44, 2015.

18. Zhang C, Wang J, Ma X, Wang W, Zhao B, Chen Y, Chen $\mathrm{C}$ and Bihl JC: ACE2-EPC-EXs protect ageing ECs against hypoxia/reoxygenation-induced injury through the miR-18a/Nox2/ROS pathway. J Cell Mol Med 22: 1873-1882, 2018.

19. Iglesias-Bartolome R, Patel V, Cotrim A, Leelahavanichkul K, Molinolo AA, Mitchell JB and Gutkind JS: mTOR inhibition prevents epithelial stem cell senescence and protects from radiation-induced mucositis. Cell Stem Cell 11: 401-414, 2012.

20. Guo Y, Li P, Gao L, Zhang J, Yang Z, Bledsoe G, Chang E, Chao $L$ and Chao J: Kallistatin reduces vascular senescence and aging by regulating microRNA-34a-SIRT1 pathway. Aging Cell 16: 837-846, 2017.

21. Lewinska A, Adamczykgrochala J, Kwasniewicz E, Deregowska A, Semik E, Zabek T and Wnuk M: Reduced levels of methyltransferase DNMT2 sensitize human fibroblasts to oxidative stress and DNA damage that is accompanied by changes in proliferation-related miRNA expression. Redox Biol 14: 20-34, 2018.

22. Gu A, Jie Y, Sun L, Zhao S, E M and You Q: RhNRG-1 $\beta$ protects the myocardium against irradiation-induced damage via the ErbB2-ERK-SIRT1 signaling pathway. Plos One 10: e0137337, 2015.

23. Livak KJ and Schmittgen TD: Analysis of relative gene expression data using real-time quantitative PCR and the 2(-Delta Delta C(T)) method. Methods 25: 402-408, 2001.

24. Crepin T, Carron C, Roubiou C, Gaugler B, Gaiffe E, Simula-Faivre D, Ferrand C, Tiberghien P, Chalopin JM, Moulin B, et al: ATG-induced accelerated immune senescence: Clinical implications in renal transplant recipients. Am J Transplant 15: 1028-1038, 2015.

25. Filippi-Chiela EC, Bueno e Silva MM, Thomé MP and Lenz G: Single-cell analysis challenges the connection between autophagy and senescence induced by DNA damage. Autophagy 11: 1099-1113, 2015

26. Ewer MS and Ewer SM: Cardiotoxicity of anticancer treatments. Nat Rev Cardiol 12: 547-558, 2015.

27. Cohen S, Liu A, Gurvitz M, Guo L, Therrien J, Laprise C, Kaufman JS, Abrahamowicz M and Marelli AJ: Exposure to low-dose ionizing radiation from cardiac procedures and malignancy risk in adults with congenital heart disease. Circulation 137: 1334-1345-2018. 
28. Dess RT, Sun Y, Matuszak MM, Sun G, Soni PD, Bazzi L, Murthy VL, Hearn JWD, Kong FM, Kalemkerian GP, et al: Cardiac events after radiation therapy: Combined analysis of prospective multicenter trials for locally advanced non-small-cell lung cancer. J Clin Oncol 35: 1395-1402, 2017.

29. Kaushik G,Spenlehauer A,Sessions AO, Trujillo AS,Fuhrmann A, Fu Z, Venkatraman V, Pohl D, Tuler J, Wang M, et al: Vinculin network-mediated cytoskeletal remodeling regulates contractile function in the aging heart. Sci Transl Med 7: 292ra299, 2015.

30. Blice-Baum AC, Zambon AC, Kaushik G, Viswanathan MC, Engler AJ, Bodmer R and Cammarato A: Modest overexpression of FOXO maintains cardiac proteostasis and ameliorates age-associated functional decline. Aging Cell 16: 93-103, 2017.

31. Dorn GW II: miR-34a and the cardiomyopathy of senescence: SALT PNUTS, SALT PNUTS! Cell Metab 17: 629-630, 2013.

32. He X, Yang A, Mcdonald DG, Riemer EC, Vanek KN, Schulte BA and Wang GY: MiR-34a modulates ionizing radiation-induced senescence in lung cancer cells. Oncotarget 8: 69797-69807, 2017.

33. Merk M, Mitchell RA, Endres S and Bucala R: D-dopachrome tautomerase 1 (D-DT or MIF-2): Doubling the MIF cytokine family. Cytokine 59: 10-17, 2012.

34. Yoshihisa Y, Rehman MU, Kondo T and Shimizu T: Role of macrophage migration inhibitory factor in heat-induced apoptosis in keratinocytes. FASEB J 30: 3870-3877, 2016.
35. von Muhlinen N, Horikawa I, Alam F, Isogaya K, Lissa D, Vojtesek B, Lane DP and Harris CC: p53 isoforms regulate premature aging in human cells. Oncogene 37: 2379-2393, 2018.

36. Zhao T, Li J and Chen AF: MicroRNA-34a induces endothelial progenitor cell senescence and impedes its angiogenesis via suppressing silent information regulator 1. Am J Physiol Endocrinol Metab 299: E110-E116, 2010.

37. Venkatachalam G, Surana U and Clément MV: Replication stress-induced endogenous DNA damage drives cellular senescence induced by a sub-lethal oxidative stress. Nucleic Acids Res 45: 10564-10582, 2017.

38. Mallette FA, Gaumont-Leclerc MF and Ferbeyre G: The DNA damage signaling pathway is a critical mediator of oncogene-induced senescence. Genes Dev 21: 43-48, 2007.

39. Purvis JE, Karhohs KW, Mock C, Batchelor E, Loewer A and Lahav G: p53 dynamics control cell fate. Science 336: 1440-1444, 2012.

40. Lin KC, Yip HK, Shao PL, Wu SC, Chen KH, Chen YT, Yang CC, Sun CK, Kao GS, Chen SY, et al: Combination of adipose-derived mesenchymal stem cells (ADMSC) and ADMSC-derived exosomes for protecting kidney from acute ischemia-reperfusion injury. Int J Cardiol 216: 173-185, 2016. 